\title{
Detection of Brain Strokes Using Microwave Tomography
}

\author{
Vanna Lisa Coli*, Pierre-Henri Tournier ${ }^{\dagger}$ Victorita Dolean ${ }^{\|}$, \\ Ibtissam El Kanfoud ${ }^{\S}$, Christian Pichot $^{\S}$, Claire Migliaccio ${ }^{\S}$, and Laure Blanc-Féraud $₫$ \\ *Dipartimento di Scienze Fisiche, Informatiche e Matematiche, UNIMORE, Italy, vannalisacoli@gmail.com \\ ${ }^{\dagger}$ UMPC, CNRS, LJLL; INRIA-Paris, EPC-Alpines Paris, France, tournier@ann.jussieu.fr \\ ‡Université Côte d'Azur, CNRS, LJAD Nice, France, victorita.dolean@unice.fr \\ $\S$ Université Côte d'Azur, CNRS, LEAT, Sophia Antipolis, France, \\ ibtissam.el-kanfoud@unice.fr, christian.pichot@unice.fr, claire.migliaccio@unice.fr \\ `Université Côte d'Azur, CNRS, I3S Sophia Antipolis, France, laure.blancferaud@cnrs.fr \\ \| University of Strathclyde, Dept of Maths and Stats, Glasgow, UK, victorita.dolean@strath.ac.uk
}

\begin{abstract}
Brain stroke is a major cause of disability and death worldwide. There are two types of stroke, ischemic or cerebral infarction ( $85 \%$ of cases) and hemorrhagic $(15 \%)$. The diagnosis must be made quickly (within 3 to 4 hours after the onset of symptoms) to determine the nature of the stroke and proceed to treatment. Recent works have shown the modification of the complex permittivity according to the nature of stroke [1] in the microwave domain. We are interested here in the detection of brain strokes using microwave tomography. We present results obtained by electromagnetic simulations coupled to a realistic noise model of measurements. The forward problem is based on a massively parallel computing using domain decomposition method, and an inverse problem based on L-BFGS algorithm with a regularization based on total variation (TV).
\end{abstract}

\section{INTRODUCTION}

Brain stroke is the leading cause of physical disability in adults in developed countries. There are two types of stroke, ischemic or cerebral infarction (85\% of cases) and hemorrhagic (15\%). Ischemic stroke is due to a clot that blocks the blood circulation. An intravenous tissue plasminogen activator (tPA) is administered within 3 hours of stroke onset. In contrast, hemorrhagic stroke is caused by rupture of a blood vessel. One understands the importance of a correct diagnosis for an appropriate treatment. Currently, the nature of stroke can only be discriminated by MRI or CT imaging. However, these bulky and expensive systems require too much infrastructure to be practiced outside the hospital. We then understand the value of a system that could be used in an ambulance, for example. In addition, during the follow-up phase of the treatment, medical imaging examinations should be repeated at regular intervals. It is particularly interesting to propose an imaging system for the treatment follow-up avoiding the transport of the patient out of the care room and presenting a safety, which is not the case of the MRI or CT-scanner. Recent work has shown the dependence of complex permittivity on the nature of stroke (ischemic or hemorrhagic) [1] in the microwave. Indeed, hemorrhagic stroke results in a 10\% increase in the complex dielectric permittivity in the stroke area, while ischemic stroke corresponds to a decrease of $10 \%$. All these elements plead for the realization of a detection system and monitoring of stroke by microwave imaging. The company EMTensor GmbH (Vienna, Austria) has developed a microwave scanner for the detection and monitoring of stroke that we have modeled and investigated the possibilities of stroke reconstruction by microwave tomography. In the first part, we present the system and the main stages of reconstruction. The second part is devoted to the results of reconstruction of ischemic stroke and hemorrhagic stroke. We conclude with a short discussion on its application as part of a clinical diagnosis.

\section{EXPERIMENTAL SYSTEM AND SIMULATIONS}

The system consists of a cylindrical metallic chamber composed of 5 rings of 32 Transmitting/Receiving antennas (Fig. 11. The antennas are ceramic $\left(\epsilon_{r}^{\text {ceramic }}=59\right)$ loaded openended waveguides. The operating frequency of the system is $0.9 \mathrm{GHz}$ to $1.8 \mathrm{GHz}$. The data acquisition cycle of the system is fully electronically controlled, allowing for a total data acquisition of about $30 \mathrm{~s}$. The imaging chamber is in horizontal position, allowing easy positioning a human head within an imaging domain. A switching matrix connected to a network
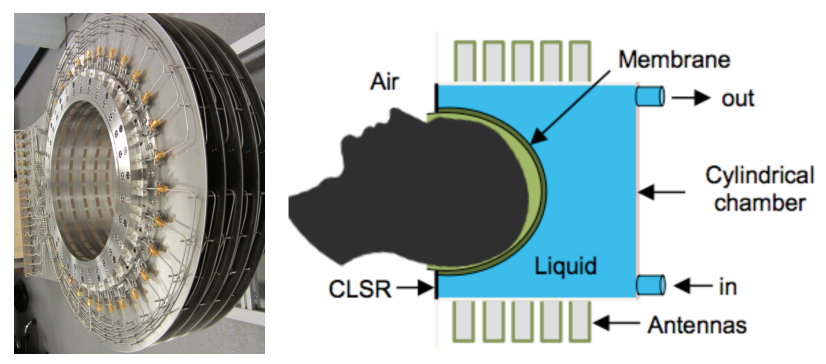

Fig. 1. Experimental system (courtesy of EMTensor GmbH).

analyzer selects the transmitting and receiving antennas. The system is potentially delivering a $160 \times 160$ matrix of $S$ parameters. The measured $S$ parameters due to the scattered field of an object under investigation are obtained by complex subtraction between two measurements with empty chamber and with the head, respectively. 


\section{NUMERICAL RESULTS}

We simulate the forward problem using a synthetic virtual brain [3]. We then corrupt the results with the model, which include the systematic and random errors using the formula

$$
S_{i j, \text { noise }}=S_{i j, \operatorname{sim}}\left(1+p\left(G_{i j}^{\mathrm{Re}}+\mathrm{i} G_{i j}^{\mathrm{Im}}\right)\right) .
$$

where $p$ represent systematic errors due to calibration and the quantities $G_{i j}$ are the realization of a random variable with Gaussian distribution, which aim to model the electronic noise of the apparatus. The signal-to-noise ratio is close to $0 \mathrm{~dB}$. The collected data are used as synthetic data to simulate the measurements. The forward problem is modeled and simulated using the open source program FreeFEM++ developed at the FreeFEM++ developed at the Université Pierre and Marie Curie, France [2] for the resolution of partial differential equations (PDE), and adapted in electromagnetism for our application [3]. Given the complexity of the problem, both in size and dielectric heterogeneity of the brain, we chose FreeFEM++ because it is designed for massively parallel computing (HPC) and incorporates a decomposition domain (DD) method. The inverse problem is based on L-BFGS algorithm (Limited-memory Broyden-Fletcher-Goldfarb-Shanno) and a smooth Total Variation (TV) regularization function [4], which aim to minimize the functional $J(\kappa)$ defined as

$$
J(\kappa)=\frac{1}{2} \sum_{j=1}^{160} \sum_{i=1}^{160}\left|\frac{S_{i j}(\kappa)}{S_{i j}^{\mathrm{em}}}-\frac{S_{i j}^{\text {meas }}}{S_{i j}^{\text {meas }, \mathrm{em}}}\right|^{2}+\alpha \int_{\Omega} \sqrt{|\nabla \kappa|^{2}+\delta}
$$

where $\kappa=k_{0}^{2} \epsilon_{r}$ is the unknown permittivity, $k_{0}$ is the wavenumber of the operating frequency in free space, $S_{i j}(\kappa)$ are the scattering coefficients computed for the permittivity $\kappa$, $S_{i j}^{\text {meas }}$ are the scattering coefficients for the head measurement,

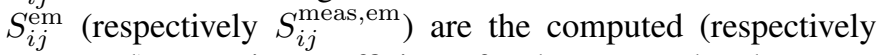
measured) scattering coefficients for the empty chamber measurement, $\alpha>0$ and $\delta>0$ are the regularization and smoothing parameter, respectively.

Fig. 2 and Fig. 3 show the cross-sections of the real and imaginary parts of the complex permittivity $\epsilon_{r}$ of the same brain, in the absence (healthy brain) or presence of an ischemic or an hemorrhagic stroke of elliptical shape, when the 3rd ring of the system is transmitting and receiving. For more visibility, we have materialized the stroke area with a white ellipse in Fig. 2 and Fig. 3
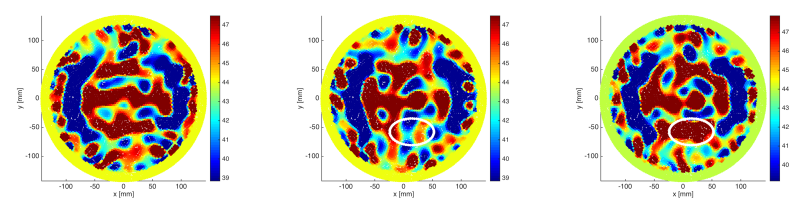

Fig. 2. Healthy brain, brain with ischemic stroke, brain with hemorrhagic stroke (real part).

Moreover, in order to better distinguish the nature of the stroke, the scale of the permittivities is adjusted so that the red corresponds to the highest values of $\epsilon_{r}$, thus close to the blood,
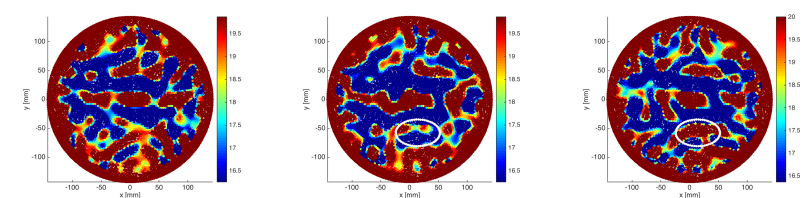

Fig. 3. Healthy brain, brain with ischemic stroke, brain with hemorrhagic stroke (imaginary part).

and the lowest correspond to blue ones. The presence of stroke is recognizable, by comparison with images of healthy brains located on the left side, in both Fig. 2 and Fig. 3 Furthermore, the nature of the stroke clearly appears in blue for the ischemic stroke and in red for the hemorrhagic one.

\section{CONCLUSION}

In this paper, we have shown the interest in microwave tomography for the detection and discrimination of the nature of a stroke. Moreover, although we were not able to work on experimental data, our method proved to be robust to noise (reconstructions with a SNR of $0 \mathrm{~dB}$ ), especially since the development of our noise model is based on exploitation of the data of a real experimental system. However, the identification of the stroke and its nature are based for the moment on the comparison of the reconstructed image of the brain with and without stroke, which remains problematic for the clinical diagnosis to the extent that the practitioner does not have, a priori, the image of the healthy brain. Research is currently continuing in this direction.

\section{ACKNOWLEDGEMENT}

This work was supported by the French National Research Agency (ANR) project MEDIMAX, as well as by the IDEX UCA JEDI with the WIMAG project. The authors would also like to thank EMTensor GmbH, Vienna, Austria, for many discussions on their experimental system.

\section{REFERENCES}

[1] S. Semenov, R. Svenson, V. Posukh, A. Nazarov, Y. Sizov, A. Bulyshev, A. Souvorov, W. Chen, J. Kassell, and G. Tatsis, "Dielectrical spectroscopy of canine myocardium during acute ischemia and hypoxia at frequency spectrum from $100 \mathrm{kHz}$ to $6 \mathrm{GHz}$, IEEE Trans. Med. Imag., vol. 21, no.6, pp. 703-707, June 2002.

[2] F. Hecht, "New development in FreeFem++", J. Numer. Math., vol. 20, no. 3-4, pp. 251-265, 2012.

[3] P. H. Tournier, M. Bonazzoli, V. Dolean, F. Rapetti, F. Hecht, F. Nataf, I. Aliferis, I. El Kanfoud, C. Migliaccio, M. de Buhan, M. Darbas, S. Semenov, and C. Pichot, Numerical Modeling and High-Speed Parallel Computing: New Perspectives on Tomographic Microwave Imaging for Brain Stroke Detection and Monitoring, IEEE Antennas Propag. Mag., vol. 59, no. 5, pp. 98-110, October 2017

[4] L. Rudin, S. Osher, E. Fatemi, "Nonlinear total variation based noise removal algorithms", Physica D., vol. 60, pp. 259-268, 1992. 